\title{
Haribabu Arthanari
}

\author{
A spin-jock's path to biomolecular dynamics with NMR, and why solitude matters.
}

W hen Shyama, the "Top Dog" in his lab, isn't happy with the data, neither is Haribabu Arthanari, a researcher at Harvard Medical School (HMS) and the Dana Farber Cancer Institute, who joined the HMS faculty in 2016. Upon arrival at HMS as a postdoctoral fellow after completing his $\mathrm{PhD}$ research at Wesleyan University, "I was more of a spin-jock," says Arthanari. He worked on teasing out cleaner electromagnetic signals from nuclear magnetic resonance spectroscopy (NMR), in which nuclear spins are probed with radio frequency pulses. His postdoctoral advisor, Gerhard Wagner, encouraged him to develop tools to query atoms in biomolecules. It led him to want to understand how molecular machines move. Arthanari's new method helps with that and can be applied to proteins, DNA or RNA. It involves ${ }^{19} \mathrm{~F}-{ }^{13} \mathrm{C}$ labeling of proteins and is called aromatic ${ }^{19} \mathrm{~F}-{ }^{13} \mathrm{C}$ TROSY,

which stands for transverse relaxationoptimized spectroscopy.

To use the new approach, biologists need not first become chemists, or change the way they make nucleic acids or proteins. They do need a ${ }^{13} \mathrm{C}$ fluorinated nucleotide or aromatic amino acid and can otherwise use their traditional method. "We want people to really use this," says Arthanari. To him, the approach is like having a new camera lens. "Now we want to start taking pictures," he says. That might involve events in the low microsecond regime that were previously not easily accessible. "It also gives us a new window into dynamics." He looks forward to using this technique to explore the many secrets even well-known molecular machines hold. Traditionally, NMR is about labeled hydrogen atoms. "Hydrogen is a lovely nucleus," he says. "I have no hate for hydrogen, but the problem with hydrogen is: it's everywhere," especially in biological systems. The new approach gives a readout only from the fluorines, which have similar qualities to hydrogen but are much more sensitive to their surroundings than hydrogen. This trait means that when labs add a second protein to the NMR tube, they get a fine-tuned look at this perturbation.

Fluorine's drawback, however, is that it relaxes quicker than hydrogen. "If you're in

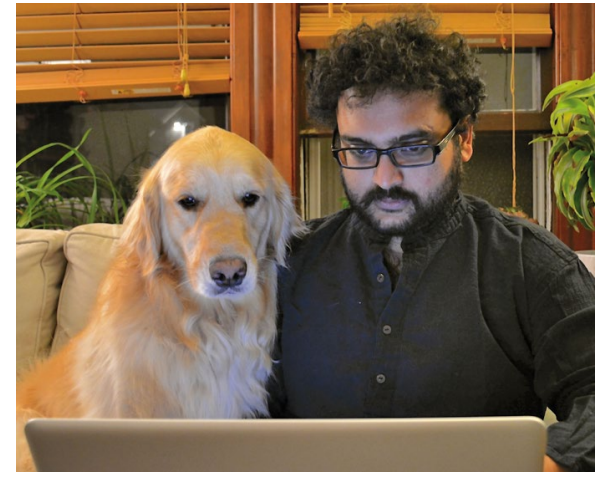

Haribabu Arthanari and Shyama

a yoga studio, relaxation is good; in NMR relaxation is bad," says Arthanari. It clutters the NMR signal, which looks a little like a photo of a crowd of marathon runners early in a race. The signals don't separate well because fast relaxation cuts data capture too short. Enter TROSY, "an old trick," which applies a kind of "spin gymnastics" to two factors that cause relaxation, chemical shift anisotropy and dipole-dipole relaxation, such that they cancel one another out. "There are two bad guys and we let them fight each other," he says. Result: the signal improves dramatically. Standard spectrometers can "pulse in fluorine and carbon," says Arthanari, but one needs the labeled precursor. "The bottleneck in this process is the labeled amino acid," he says. It must be labeled with both ${ }^{13} \mathrm{C}$ and ${ }^{19} \mathrm{~F}$. No vendor sold this, but he and his team have cracked the synthesis problem. Some vendors have now indicated to Arthanari their interest in offering these amino acids, which would grow the method's user base. The fluorinated protein is made in bacteria, leveraging a method from University of Toronto's Scott Prosser and others. With a chemical, the bacterial shikimate pathway, through which aromatic amino acids are synthesized, is shut down. Protein synthesis is disrupted. To survive, the cells take up fluorinated amino acid and make a labeled protein.

Paraphrasing Louis Pasteur, Arthanari says that discovery calls for a prepared mind. His preparation happens in discussions with his team members and with colleagues around the globe. One must be prepared for the "jumps" nature presents. "The more your mind is prepared to explore these jumps, that is where the important things happen," he says. Arthanari began thinking more about fluorinated labels after a visit to Angela Gronenborn's lab. Ideas and calculations lead to discovery and then it takes rigorous development to assure an approach works under many conditions. Chemist and structural biologist Angela Gronenborn at the University of Pittsburgh School of Medicine calls Arthanari "highly creative in his approaches and refreshingly unconventional in his view of the scientific problems that need to be solved." She met him in Berlin, where she was a visiting Einstein Foundation fellow. At her lab, he settled down at the spectrometer, where a fluorinated protein was in the NMR tube. "He played the spectrometer like a pianoskillfully and with feeling," she says. "It was a delight to watch." His new fluorine NMR experiments fill her with joy, as did their cycling through Berlin and scientific discussions.

\section{"I was more of a spin-jock."}

When he is not in the lab, Arthanari travels. "Travel to where I can have some solitude is something that I love," he says. He's been to the Amazon, to Patagonia and to Svalbard, near the North Pole. "Go there, all you have is the basic necessities and a lot of time to think," he says. When he travels, Shyama has to stay at home. She would likely run to say hi to one of Svalbard's many polar bears, he says. "I don't want to think what the polar bear would say."

\section{Vivien Marx}

Published online: 18 March 2019 https://doi.org/10.1038/s41592-019-0362-6

\section{References}

Boeszoermenyi, A. et al. Aromatic ${ }^{19} \mathrm{~F}-{ }^{13} \mathrm{C}$ TROSY: a background-free approach to probe biomolecular structure, function, and dynamics. Nat. Methods https://doi.org/10.1038/s41592-019-0334-x (2019). 